\title{
Aceptación de los docentes al modelo curricular por competencias en la Universidad Nacional José Faustino Sánchez Carrión - 2016
}

\author{
Acceptance of teachers to the curricular model by competencies at the \\ National University José Faustino Sánchez Carrión - 2016 \\ José Natividad Farroñan Santiesteban', Santiago Pedro Ravines Miranda', \\ Delman Yoplack Zumaeta ${ }^{2}$, Félix Gil Caro Soto ${ }^{2}$
}

\section{RESUMEN}

Objetivo: Determinar el grado de aceptación al modelo curricular por competencias por parte de los docentes en la Universidad Nacional José Faustino Sánchez Carrión. Materiales y métodos: Como instrumento se aplicó un cuestionario a 129 docentes de la Universidad Nacional "José Faustino Sánchez Carrión" para determinar el grado de aceptación al nuevo plan curricular por competencias. Materiales: Escritorio, Computadora, Impresora, software estadístico SPSS para Windows v.20, cuestionarios. Metodología: El estudio corresponde a una investigación de tipo descriptivo, tuvo por finalidad describir un fenómeno o una situación en una circunstancia temporal-espacial determinada. Hoy en día las universidades con la finalidad de afrontar los desafíos de la globalización, están en proceso de implementar planes curriculares por competencias, frente a esto los docentes deben adecuarse al nuevo modelo de manera obligatoria lo que genera cierta actitud reacia por parte de algunos docentes sobre todo en los docentes antiguos que estaban acostumbrados a trabajar con el plan curricular por objetivos; la Universidad Nacional "José Faustino Sánchez Carrión" no es ajena a los cambios que requiere la educación superior a nivel mundial. Conclusiones: Los docentes de la Universidad Nacional "José Faustino Sánchez Carrión" que dictan los primeros ciclos es decir con el Plan Curricular por Competencias están aceptando este nuevo modelo curricular.

Palabras claves: plan curricular, modelo curricular por competencias.

\begin{abstract}
Objective: To determine the degree of acceptance to the curricular model by competences by the teachers at the National University José Faustino Sánchez Carrión. Material And Methods: As an instrument, a questionnaire was applied to 129 teachers of the National University "Jose Faustino Sanchez Carrion" to determine the degree of acceptance to the new curricular plan by competencies. Materials: Desktop, Computer, Printer, SPSS Statistical Software for Windows v.20, questionnaires. Methodology: The study corresponds to a research of descriptive type, its purpose was to describe a phenomenon or a situation in a specific temporal-spatial circumstance.Nowadays the universities with the purpose of addressing the challenges of globalization, are in the process of implementing curricular plans by competencies, as opposed to this teachers must be adapted to the new model on a compulsory basis which generates a certain reluctance on the part of some teachers especially in the old teachers who were accustomed to working with the curricular plan by objectives; the National University "Jose Faustino Sanchez Carrion" is not unaware of the changes that are needed in the superior education worldwide. Conclusions: The teachers of the National University "José Faustino Sánchez Carrión" that dictate the first cycles it means with the Curricular Plan by Competencies are accepting this new curricular model.
\end{abstract}

Key words: Curricular plan, curriculum model for competencies.

\footnotetext{
${ }^{1}$ Docente. Facultad de Ciencias. Universidad Nacional José Faustino Sánchez Carrión. Lima - Perú.

${ }^{2}$ Docente. Facultad de Ciencias Empresariales. Universidad Nacional José Faustino Sánchez Carrión
} 


\section{INTRODUCCIÓN}

En la actualidad los procesos de globalización generan un gran impacto en la educación superior, pues cambian la manera de producir conocimiento, su aplicación y difusión. Las universidades como instituciones líderes de la educación superior y la promoción de la investigación cumplen un papel crucial en la gestión del servicio educativo y el conocimiento para afrontar los grandes desafíos que conlleva la globalización. De ahí la necesidad de adaptar sus roles y desarrollaren sus estudiantes competencias que les permitan anticiparse y responder a los cambios en los ámbitos local, regional e internacional. Los estudiantes aspiran hoy a carreras que les permitan emplearse rápidamente.

La globalización de la educación superior, según Deepak Nayyar (2012), conlleva efectos significativos para las comunidades locales y los países: ha tomado las universidades, ha incrementado sustancialmente la movilidad académica, ha expandido las corporaciones trasnacionales y se ha convertido en un fenómeno de alcance mundial.

Según Campero (2008), hoy se exige a las instituciones educativas del nivel superior formar personas competentes, lo que hace que la formación deba apuntar al vínculo con el campo del desempeño profesional y la participación social, pues la sola actividad académica y la acumulación de conocimientos o de información no garantizan el dominio y la experticia en competencias profesionales, menos aún si esa formación continúa siendo de carácter asignaturista o disciplinar, aislada, sin un eje integrador que permita comprender la complejidad de interrelaciones y sistemas propios del ejercicio en la vida del profesional en su contexto.

\section{MATERIAL Y MÉTODO}

Como método se aplicó un cuestionario a 129 docentes de la Universidad Nacional "José Faustino Sánchez Carrion" para determinar el grado de aceptación al nuevo plan curricular por competencias.

\section{Materiales:}

Escritorio, computadora, impresora, software estadístico SPSS para Windows v.20, Cuestionarios.

\section{Metodología:}

El estudio corresponde a una investigación de tipo descriptivo, tuvo por finalidad describir un fenómeno o una situación en una circunstancia temporal-espacial determinada.

\section{Instrumentos utilizados:}

Test: Se recolectó información acerca del rendimiento académico de los alumnos de la Facultad de Ciencias Empresariales.

El Cuestionario: Se elaboró un cuestionario para conocer al grado de aceptación.

\section{Las técnicas utilizadas}

Encuesta: Se recolectó los datos sobre la opinión de cada docente de forma personal y directa y los ítem fueron afirmaciones con alternativas precisas de respuesta. La población estuvo constituida por los docentes de las diferentes facultades de la Universidad Nacional "José Faustino Sánchez Carrión" que dictan clases en el ciclo académico 2016-II. El tamaño de la muestra fue de 129, considerada una muestra aleatoria con tamaño grande, siendo adecuado y representativo en la recolección de datos.

En el análisis estadístico se usaron técnicas estadísticas de procesamiento y análisis de datos tales como tablas de frecuencia, y además para contrastar las hipótesis se utilizó la estadística Chi cuadrado.

\section{RESULTADOS}

Las respuestas son variables dicotómicas, es decir tienen dos únicas categorías como, por ejemplo, presencia/ausencia o mujer/hombre, entonces tenemos una tabla de contingencia de $2 \times 2$, y se usa una corrección del test 2 , la corrección de Yates (1934) o el test de Fisher (1922).

Tabla 1: Relación entre acepta usted el modelo curricular por competencias y cree Ud., que el modelo curricular por competencias es un modelo rígido.

Medir el grado de aceptación de los docentes de la Universidad José Faustino Sánchez Carrión 2016 -UNJFSC.

\begin{tabular}{|c|c|c|c|}
\hline \multicolumn{4}{|c|}{ Estadísticas de la Regresión } \\
\hline \multicolumn{3}{|c|}{ Coeficiente de correlación múltiple } & 0.383 \\
\hline \multicolumn{3}{|c|}{ Coeficiente de determinación $\mathrm{R}^{\wedge} 2$} & 0.147 \\
\hline \multicolumn{3}{|l|}{$\mathrm{R}^{\wedge} 2$ ajustado } & 0.119 \\
\hline \multicolumn{3}{|l|}{ Error típico } & 0.342 \\
\hline \multicolumn{3}{|l|}{ Observaciones } & 129 \\
\hline \multirow[t]{2}{*}{$\begin{array}{l}\text { Es un modelo } \\
\text { Rígido }\end{array}$} & \multicolumn{2}{|c|}{$\begin{array}{l}\text { Acepta el Modelo Curricular por } \\
\text { Competencia } \\
\end{array}$} & \multirow[t]{2}{*}{ Total } \\
\hline & Si & No & \\
\hline Si & 31 & 15 & 46 \\
\hline No & 78 & 5 & 83 \\
\hline Total & 109 & 20 & 129 \\
\hline
\end{tabular}

\begin{tabular}{lccccc}
\hline ANÁLISIS DE VARIANZA & & & \\
\hline & $\begin{array}{c}\text { Grados de } \\
\text { libertad }\end{array}$ & $\begin{array}{c}\text { Suma de } \\
\text { cuadrados }\end{array}$ & $\begin{array}{c}\text { Promedio de } \\
\text { los cuadrados }\end{array}$ & F & $\begin{array}{c}\text { Valor critico } \\
\text { de F }\end{array}$ \\
\hline Regresión & 1 & 0.623 & 0.623 & 5.340 & $\mathbf{0 . 0 2 8}$ \\
Residuos & 128 & 3.619 & 0.117 & & \\
Total & 129 & 4.242 & & & \\
\hline
\end{tabular}




\begin{tabular}{lcccccc}
\hline Coeficientes & $\begin{array}{c}\text { Error } \\
\text { tipico }\end{array}$ & $\begin{array}{c}\text { Estadístico } \\
\mathbf{t}\end{array}$ & Probabilidad & $\begin{array}{c}\text { Inferior } \\
\mathbf{9 5 \%}\end{array}$ & $\begin{array}{c}\text { Superior } \\
\mathbf{9 5 \%}\end{array}$ \\
\hline Intercepción & 0.333 & 0.0 & 3.380 & 0.002 & 0.13 & 0.534 \\
& & 99 & & & 2 & \\
Modelo & -0.286 & 0.1 & -2.311 & 0.028 & - & - \\
Rigido & & 24 & & & 0.53 & 0.034 \\
& & & & & & \\
\hline
\end{tabular}

La prueba de Fisher demuestra que si existe relación entre aceptar el modelo curricular por competencias y que el modelo curricular por competencias es un modelo rígido.

Se observa que el valor critico de $\mathrm{F}$ ( $\mathrm{p}$-valué) es menor al $\alpha=0.05$

Con una correlación baja de 0.383

Tabla 2: Relación entre acepta usted el modelo curricular por competencias y cree Ud., que el modelo curricular por competencias con llevara al aprendizaje de los estudiantes.

Medir el grado de Aceptación de los Docentes de la Universidad José Faustino Sánchez Carrión 2016 -UNJFSC

\begin{tabular}{cccc}
\hline \multirow{2}{*}{$\begin{array}{c}\text { Conllevara al } \\
\text { aprendizaje de } \\
\text { los estudiantes }\end{array}$} & $\begin{array}{c}\text { Acepta el Modelo Curricular por } \\
\text { Competencia }\end{array}$ & Total \\
\cline { 2 - 3 } & $\mathbf{S i}$ & No & \\
\hline $\mathbf{S i}$ & 74 & 0 & 74 \\
No & 35 & 20 & 55 \\
Total & 109 & 20 & 129 \\
\hline \multicolumn{4}{c}{ Estadísticas de la regresión } \\
\hline Coeficiente de correlación múltiple \\
Coeficiente de determinación R^2 \\
R^2 ajustado & 0.492 \\
Error típico & 0.242 \\
Observaciones & 0.218 \\
\hline
\end{tabular}

\begin{tabular}{lccccc}
\hline ANÁLISIS DE VARIANZA & & & \\
\hline $\begin{array}{c}\text { Grados de } \\
\text { libertad }\end{array}$ & $\begin{array}{c}\text { Suma de } \\
\text { cuadrados }\end{array}$ & $\begin{array}{c}\text { Promedio de } \\
\text { los cuadrados }\end{array}$ & $\begin{array}{c}\text { F } \\
\text { Valor critico } \\
\text { de F }\end{array}$ \\
\hline Regresión & 1 & 1.028 & 1.028 & 9.916 & $\mathbf{0 . 0 0 4}$ \\
Residuos & 128 & 3.214 & 0.104 & & \\
Total & 129 & 4.242 & & & \\
\hline
\end{tabular}

\begin{tabular}{lcccccc}
\hline Coeficientes & $\begin{array}{c}\text { Error } \\
\text { tipico }\end{array}$ & $\begin{array}{c}\text { Estadístico } \\
\mathbf{t}\end{array}$ & Probabilidad & $\begin{array}{c}\text { Inferior } \\
\mathbf{9 5 \%}\end{array}$ & $\begin{array}{c}\text { Superior } \\
\mathbf{9 5 \%}\end{array}$ \\
\hline Intercepción & 0.000 & 0.0 & 0.000 & 1.000 & & 0.151 \\
& & 74 & & & 0.15 & 1 \\
& & 0.1 & 3.149 & 0.004 & 0.12 & 0.588 \\
$\begin{array}{l}\text { Conllevara al } \\
\text { aprendiaze de } \\
\text { los estudiantes }\end{array}$ & 0.357 & 0.13 & & & 6 & \\
\hline
\end{tabular}

La prueba de Fisher demuestra que si existe relación entre aceptar el modelo curricular por competencias y que el modelo curricular por competencias con llevara al aprendizaje de los estudiantes.

Se observa que el valor critico de $F$ ( $p$-valué) es menor al $\alpha=0.05$
Con una correlación baja de 0.492 .

Tabla 3: Relación entre acepta usted el modelo curricular por competencias y cree Ud., que el modelo curricular por competencias potencia el aprendizaje para lograr el desarrollo personal integral. Tabla 3

Medir el grado de aceptación de los docentes de la Universidad José Faustino Sánchez Carrión 2016 -UNJFSC.

\begin{tabular}{cccc}
\hline $\begin{array}{c}\text { Lograr el } \\
\text { desarrollo } \\
\text { personal } \\
\text { integral }\end{array}$ & $\begin{array}{c}\text { Acepta el Modelo Curricular por } \\
\text { Competencia }\end{array}$ & Total \\
\cline { 2 - 3 } & Si & No & \\
\hline Si & 86 & 3 & 89 \\
No & 23 & 17 & 40 \\
Total & 109 & 20 & 129 \\
\hline
\end{tabular}

\begin{tabular}{lc}
\hline \multicolumn{2}{c}{ Estadísticas de la regresión } \\
\hline Coeficiente de correlación múltiple & 0.457 \\
Coeficiente de determinación $\mathrm{R}^{\wedge} 2$ & 0.209 \\
$\mathrm{R}^{\wedge} 2$ ajustado & 0.183 \\
Error típico & 0.329 \\
Observaciones & 33 \\
\hline
\end{tabular}

\begin{tabular}{|c|c|c|c|c|c|c|c|c|}
\hline \multicolumn{9}{|c|}{ ANÁLISIS DE VARIANZA } \\
\hline & $\begin{array}{l}\text { Grados de } \\
\text { libertad }\end{array}$ & \multicolumn{2}{|c|}{$\begin{array}{c}\text { Suma de } \\
\text { cuadrados }\end{array}$} & \multicolumn{2}{|c|}{$\begin{array}{l}\text { Promedio de } \\
\text { los cuadrados }\end{array}$} & $\mathrm{F}$ & \multicolumn{2}{|c|}{$\begin{array}{c}\text { Valor critico } \\
\text { de } F\end{array}$} \\
\hline Regresión & 1 & & 886 & & 0.886 & 8.182 & & 0.008 \\
\hline Residuos & 128 & & 357 & & 0.108 & & & \\
\hline \multirow[t]{2}{*}{ Total } & 129 & & 242 & & & & & \\
\hline & oeficientes & $\begin{array}{l}\text { Error } \\
\text { tipico }\end{array}$ & $\begin{array}{r}\text { Estadis } \\
t\end{array}$ & & Probabilidad & & $\begin{array}{l}\text { ferior } \\
95 \%\end{array}$ & $\begin{array}{c}\text { Superior } \\
95 \%\end{array}$ \\
\hline Intercepción & 0.043 & $\begin{array}{l}0.0 \\
69\end{array}$ & 0.634 & & 0.531 & & 0.09 & 0.183 \\
\hline $\begin{array}{l}\text { Lograr el } \\
\text { desarrollo } \\
\text { personal integral }\end{array}$ & 0.357 & $\begin{array}{l}0.1 \\
25\end{array}$ & 2.869 & & 0.008 & & $\begin{array}{c}6 \\
0.10 \\
2\end{array}$ & 0.611 \\
\hline
\end{tabular}

La prueba de Fisher demuestra que si existe relación entre aceptar el modelo curricular por competencias y que el modelo curricular por competencias potencia el aprendizaje para lograr el desarrollo personal integral..

Se Observa que el valor critico de $F$ ( $p$-valué) es menor al $\alpha=0.05$

Con una correlación baja de 0.457 .

Tabla 4: Relación entre acepta usted el modelo curricular por competencias y cree Ud., que para el cambio pedagógico es necesario implementar la universidad con tecnología moderna.

Medir el grado de aceptación de los docentes de la Universidad José Faustino Sánchez Carrión 2016 -UNJFSC. 


\begin{tabular}{|c|c|c|c|c|c|c|}
\hline \multirow{2}{*}{\multicolumn{2}{|c|}{$\begin{array}{c}\text { Implementar la } \\
\text { universidad con } \\
\text { tecnología } \\
\text { moderna } \\
\end{array}$}} & \multicolumn{4}{|c|}{$\begin{array}{l}\text { Acepta el Modelo Curricular por } \\
\text { Competencia }\end{array}$} & \multirow[t]{2}{*}{ Total } \\
\hline & & \multicolumn{2}{|r|}{$\mathrm{Si}$} & \multicolumn{2}{|r|}{ No } & \\
\hline Si & & \multicolumn{2}{|r|}{94} & \multicolumn{2}{|r|}{15} & 109 \\
\hline No & & \multicolumn{2}{|r|}{16} & \multicolumn{2}{|r|}{4} & 20 \\
\hline Total & & \multicolumn{2}{|c|}{110} & \multicolumn{2}{|r|}{19} & 129 \\
\hline \multicolumn{7}{|c|}{ Estadísticas de la regresión } \\
\hline \multicolumn{6}{|c|}{ Coeficiente de correlación múltiple } & 0.057 \\
\hline \multicolumn{6}{|c|}{ Coeficiente de determinación $\mathrm{R}^{\wedge} 2$} & 0.003 \\
\hline \multicolumn{6}{|c|}{$\mathrm{R}^{\wedge} 2$ ajustado } & -0.029 \\
\hline \multicolumn{6}{|l|}{ Error típico } & 0.369 \\
\hline \multicolumn{6}{|c|}{ Observaciones } & 129 \\
\hline \multicolumn{7}{|c|}{ ANÁLISIS DE VARIANZA } \\
\hline \multicolumn{2}{|c|}{$\begin{array}{l}\text { Grados de } \\
\text { libertad }\end{array}$} & \multicolumn{2}{|c|}{$\begin{array}{cc}\text { Suma de } & F \\
\text { cuadrados } & \text { lo }\end{array}$} & $\begin{array}{l}\text { Promedio de } \\
\text { os cuadrados }\end{array}$ & $\mathrm{F} \mathrm{Va}$ & $\begin{array}{l}\text { Valor critico } \\
\text { de F }\end{array}$ \\
\hline Regresión & 1 & & .014 & 0.014 & 0.102 & 0.752 \\
\hline Residuos & 128 & & .229 & 0.136 & & \\
\hline \multirow[t]{2}{*}{$\underline{\text { Total }}$} & 129 & & .242 & & & \\
\hline & ficientes & $\begin{array}{l}\text { Error } \\
\text { tipico }\end{array}$ & $\begin{array}{c}\text { Estadístico } \\
\mathrm{t}\end{array}$ & Probabilidad & Id $\begin{array}{r}\text { Inferio } \\
95 \%\end{array}$ & $\begin{array}{cc}\text { or } & \text { Superior } \\
95 \%\end{array}$ \\
\hline Intercepción & 0.143 & $\frac{0.0}{70}$ & 2.047 & 0.049 & $\stackrel{0.00}{1}$ & 0.285 \\
\hline $\begin{array}{l}\text { Implementar la } \\
\text { universidad con } \\
\text { techologia moderna }\end{array}$ & 0.057 & $\begin{array}{l}0.1 \\
79\end{array}$ & 0.319 & 0.752 & 0.30 & 0.423 \\
\hline
\end{tabular}

La prueba de Fisher demuestra que no existe relación entre aceptar el modelo curricular por competencias y que para el cambio pedagógico es necesario implementar la universidad con tecnología moderna.

Se observa que el valor critico de $F$ ( $p$-valué) es mayor al $\alpha=0.05$

No hay correlación, es de 0.057 (tiende a cero).

Tabla 5: Relación entre acepta usted el modelo curricular por competencias y a hecho Ud., en la actualidad algún cambio en su práctica docente.

Medir el grado de aceptación de los docentes de la Universidad José Faustino Sánchez Carrión 2016 -UNJFSC.

\begin{tabular}{cccc}
\hline $\begin{array}{c}\text { En la actualidad } \\
\text { algún cambio en } \\
\text { su práctica } \\
\text { docente }\end{array}$ & $\begin{array}{c}\text { Acepta el Modelo Curricular por } \\
\text { Competencia }\end{array}$ & Total \\
\cline { 2 - 3 } & $\mathbf{S i}$ & No & \\
\hline $\mathrm{Si}$ & 82 & 15 & 97 \\
No & 27 & 5 & 32 \\
Total & 109 & 20 & $\mathbf{1 2 9}$ \\
\hline
\end{tabular}

\begin{tabular}{lc}
\hline \multicolumn{2}{c}{ Estadísticas de la regresión } \\
\hline Coeficiente de correlación múltiple & 0.042 \\
Coeficiente de determinación $\mathrm{R}^{\wedge} 2$ & 0.002 \\
$\mathrm{R}^{\wedge} 2$ ajustado & -0.030 \\
Error típico & 0.370 \\
Observaciones & 129 \\
\hline
\end{tabular}

\begin{tabular}{lccccc}
\hline ANÁLISIS DE VARIANZA & & & \\
\hline & $\begin{array}{c}\text { Grados de } \\
\text { libertad }\end{array}$ & $\begin{array}{c}\text { Suma de } \\
\text { cuadrados }\end{array}$ & $\begin{array}{c}\text { Promedio de } \\
\text { los cuadrados }\end{array}$ & F & $\begin{array}{c}\text { Valor critico } \\
\text { de F }\end{array}$ \\
\hline Regresión & 1 & 0.014 & 0.014 & 0.102 & $\mathbf{0 . 7 5 2}$ \\
Residuos & 128 & 4.229 & 0.136 & & \\
Total & 129 & 4.242 & & & \\
\hline
\end{tabular}

\begin{tabular}{|c|c|c|c|c|c|c|}
\hline & Coeficientes & $\begin{array}{l}\text { Error } \\
\text { tipico }\end{array}$ & $\begin{array}{c}\text { Estadístico } \\
t\end{array}$ & Probabilidad & $\begin{array}{l}\text { Inferior } \\
95 \%\end{array}$ & $\begin{array}{c}\text { Superior } \\
95 \%\end{array}$ \\
\hline Intercepción & 0.160 & $\begin{array}{l}0.0 \\
74\end{array}$ & 2.164 & 0.038 & $\begin{array}{c}0.00 \\
9\end{array}$ & 0.311 \\
\hline $\begin{array}{l}\text { Algún cambio } \\
\text { en su práctica } \\
\text { docente }\end{array}$ & -0.035 & $\begin{array}{l}0.1 \\
50\end{array}$ & -0.233 & 0.817 & $\begin{array}{c}0.34 \\
1\end{array}$ & 0.271 \\
\hline
\end{tabular}

La prueba de Fisher demuestra que no existe relación entre aceptar el modelo curricular por competencias y que en la actualidad a hecho algún cambio en su práctica docente..

Se observa que el valor critico de $F$ ( $p$-valué) es mayor al $\alpha=0.05$

No hay correlación, es de 0.042 (tiende a cero).

Tabla 6: Relación entre acepta usted el modelo curricular por competencias y cree Ud, que el modelo curricular por competencias se ajusta a la realidad en el mundo globalizado.

Medir el grado de aceptación de los docentes de la Universidad José Faustino Sánchez Carrión 2016 -UNJFSC

\begin{tabular}{lccc}
\hline $\begin{array}{l}\text { Se ajusta a la } \\
\text { realidad en el } \\
\text { mundo } \\
\text { globalizado }\end{array}$ & $\begin{array}{c}\text { Acepta el Modelo Curricular por } \\
\text { Competencia }\end{array}$ & Total \\
\cline { 2 - 3 } & $\mathbf{S i}$ & No & \\
\hline Si & 70 & 20 & 70 \\
No & 39 & 59 \\
Total & 109 & 120 \\
\hline & Estadísticas de la regresión & \\
\hline & 20 & 0.463 \\
\hline Coeficiente de correlación múltiple & 0.214 \\
Coeficiente de determinación $\mathrm{R}^{\wedge} 2$ & 0.189 \\
$\mathrm{R}^{\wedge} 2$ ajustado & 0.328 \\
Error típico & 129 \\
Observaciones & & \\
\hline
\end{tabular}

\begin{tabular}{lccccc}
\hline ANÁLISIS DE VARIANZA & & & \\
\hline $\begin{array}{c}\text { Grados de } \\
\text { libertad }\end{array}$ & $\begin{array}{c}\text { Suma de } \\
\text { cuadrados }\end{array}$ & $\begin{array}{c}\text { Promedio de } \\
\text { los cuadrados }\end{array}$ & F & $\begin{array}{c}\text { Valor critico } \\
\text { de F }\end{array}$ \\
\hline Regresión & 1 & 0.909 & 0.909 & 8.455 & $\mathbf{0 . 0 0 7}$ \\
Residuos & 31 & 3.333 & 0.108 & & \\
Total & 32 & 4.242 & & &
\end{tabular}




\begin{tabular}{lcccccc}
\hline Coeficientes & $\begin{array}{c}\text { Error } \\
\text { tipico }\end{array}$ & $\begin{array}{c}\text { Estadístico } \\
\mathbf{t}\end{array}$ & Probabilidad & $\begin{array}{c}\text { Inferior } \\
\mathbf{9 5 \%}\end{array}$ & $\begin{array}{c}\text { Superior } \\
\mathbf{9 5 \%}\end{array}$ \\
\hline Intercepción & 0.000 & 0.0 & 0.000 & 1.000 & -0.158 & 0.15 \\
& 77 & & 0.007 & 0.100 & 0.56 \\
\begin{tabular}{l}
$\begin{array}{l}\text { Se ajusta a la } \\
\text { realidad en el } \\
\text { mundo globalizado }\end{array}$ \\
\hline
\end{tabular} & 0.333 & 0.1 & 2.908 & 0.007 & & 7 \\
\hline
\end{tabular}

La prueba de Fisher demuestra que si existe relación entre aceptar el modelo curricular por competencias y que el modelo curricular por competencias se ajusta a la realidad en el mundo globalizado.

Se observa que el valor critico de $F$ ( $p$-valué) es menor al $\alpha=0.05$

Con una correlación baja de 0.463 .

Tabla 7: Relación entre acepta usted el modelo curricular por competencias y cree Ud., que el modelo curricular por competencias motiva para que el estudiante logre un mejor aprendizaje.

Medir el grado de aceptación de los docentes de la Universidad José Faustino Sánchez Carrión 2016 -UNJFSC.

\begin{tabular}{lccc}
\hline $\begin{array}{c}\text { Motiva para que } \\
\text { el estudiante } \\
\begin{array}{l}\text { logre un mejor } \\
\text { aprendizaje }\end{array}\end{array}$ & $\begin{array}{c}\text { Acepta el Modelo Curricular por } \\
\text { Competencia }\end{array}$ & Total \\
\cline { 2 - 3 } & $\mathbf{S i}$ & No & \\
\hline $\mathrm{Si}$ & 86 & 0 & 86 \\
$\mathrm{No}$ & 23 & 20 & 43 \\
Total & 109 & 20 & 129 \\
\hline \multicolumn{3}{c}{ Estadísticas de la Regresión } \\
\hline Coeficiente de correlación múltiple \\
Coeficiente de determinación R^2 \\
R^2 ajustado & 0.598 \\
Error típico & 0.357 \\
Observaciones & 0.336 \\
\hline
\end{tabular}

\begin{tabular}{lccccc}
\hline \multicolumn{7}{c}{ ANÁLISIS DE VARIANZA } \\
$\begin{array}{l}\text { Grados de } \\
\text { libertad }\end{array}$ & $\begin{array}{c}\text { Suma de } \\
\text { cuadrados }\end{array}$ & $\begin{array}{c}\text { Promedio de } \\
\text { los cuadrados }\end{array}$ & F & $\begin{array}{c}\text { Valor critico } \\
\text { de F }\end{array}$ \\
\hline Regresión & 1 & 1.515 & 1.515 & 17.222 & $\mathbf{0 . 0 0 0}$ \\
Residuos & 128 & 2.727 & 0.088 & & \\
Total & 129 & 4.242 & & & \\
\hline
\end{tabular}

\begin{tabular}{lcccccc}
\hline Coeficientes & $\begin{array}{c}\text { Error } \\
\text { tipico }\end{array}$ & $\begin{array}{c}\text { Estadístico } \\
\mathbf{t}\end{array}$ & Probabilidad & $\begin{array}{c}\text { Inferior } \\
\mathbf{9 5 \%}\end{array}$ & $\begin{array}{c}\text { Superior } \\
\mathbf{9 5 \%}\end{array}$ \\
\hline Intercepción 0.000 & 0.063 & 0.000 & 1.000 & -0.129 & 0.129 \\
$\begin{array}{l}\text { Motiva para que } \\
\text { el estudiante logre } \\
\text { un mejor } \\
\text { aprendizaje }\end{array}$ & 0.455 & 4.150 & 0.000 & 0.231 & 0.678 \\
\hline
\end{tabular}

La Prueba de Fisher demuestra que si existe relación entre aceptar el modelo curricular por competencias y que el modelo curricular por competencias motiva para que el estudiante logre un mejor aprendizaje.

Se observa que el valor critico de $F$ ( $p$-valué) es menor al $\alpha=0.05$

Big Bang Faustiniano 2017; 6(1): 9 - 14
Con una correlación alta de 0.598 .

Tabla 8: Relación entre acepta usted el modelo curricular por competencias y esta Ud., actualizado para desempeñar con calidad en la docencia.

Medir el grado de aceptación de los docentes de la Universidad José Faustino Sánchez Carrión 2016 -UNJFSC

\begin{tabular}{cccc}
\hline $\begin{array}{c}\text { Actualizado para } \\
\text { desempeñar con } \\
\text { calidad en la } \\
\text { docencia }\end{array}$ & $\begin{array}{c}\text { Acepta el Modelo Curricular por } \\
\text { Competencia }\end{array}$ & Total \\
\cline { 2 - 3 } & $\mathbf{S i}$ & No & \\
\hline $\mathbf{S i}$ & 94 & 15 & 109 \\
No & 16 & 4 & 20 \\
Total & 110 & 19 & $\mathbf{1 2 9}$ \\
\hline \multicolumn{3}{c}{ Estadísticas de la regresión } \\
\hline Coeficiente de correlación múltiple & 0.057 \\
Coeficiente de determinación R^2 & 0.003 \\
$\mathrm{R}^{\wedge 2}$ ajustado & -0.029 \\
Error típico & 0.369 \\
Observaciones & 129 \\
\hline
\end{tabular}

\begin{tabular}{|c|c|c|c|c|c|c|}
\hline \multicolumn{7}{|c|}{ ANÁLISIS DE VARIANZA } \\
\hline & $\begin{array}{c}\text { Grados de } \\
\text { libertad }\end{array}$ & \multicolumn{2}{|c|}{$\begin{array}{c}\text { Suma de } \\
\text { cuadrados }\end{array}$} & $\begin{array}{l}\text { Promedio de } \\
\text { los cuadrados }\end{array}$ & $\mathrm{F}$ & $\begin{array}{c}\text { Valor critico } \\
\text { de F }\end{array}$ \\
\hline Regresión & 1 & & 14 & 0.014 & 0.102 & 0.752 \\
\hline Residuos & 31 & & 229 & 0.136 & & \\
\hline \multirow[t]{2}{*}{ Total } & 32 & & 242 & & & \\
\hline & oeficientes & $\begin{array}{l}\text { Error } \\
\text { tipico }\end{array}$ & $\begin{array}{c}\text { Estadístico } \\
\mathrm{t}\end{array}$ & Probabilidad & $\begin{array}{c}\text { Inferior } \\
95 \%\end{array}$ & $\begin{array}{c}\text { Superior } \\
95 \%\end{array}$ \\
\hline Intercepción & 0.143 & $\begin{array}{l}0.0 \\
70\end{array}$ & 2.047 & 0.049 & 0.00 & 0.285 \\
\hline $\begin{array}{l}\text { Actualizado para } \\
\text { desempeñar con } \\
\text { calidad en la } \\
\text { docencia }\end{array}$ & $\begin{array}{ll}\mathrm{a} & 0.057 \\
\text { n } & \end{array}$ & $\begin{array}{l}0.1 \\
79\end{array}$ & 0.319 & 0.752 & 0.30 & 0.423 \\
\hline
\end{tabular}

La prueba de Fisher demuestra que no existe relación entre aceptar el modelo curricular por competencias y se encuentra actualizado para desempeñar con calidad en la docencia.

Se observa que el valor critico de $F$ ( $p$-valué) es mayor al $\alpha=0.05$

No hay correlación, es de 0.057 (tiende a cero).

Tabla 9: Relación entre acepta usted el modelo curricular por competencias y cree Ud., que el modelo curricular por competencias responde a las exigencias del mercado laboral.

Medir el grado de aceptación de los docentes de la Universidad José Faustino Sánchez Carrión 2016-UNJFSC

\begin{tabular}{cccc}
\hline $\begin{array}{c}\text { Responde a las } \\
\text { exigencias del } \\
\text { mercado laboral }\end{array}$ & $\begin{array}{c}\text { Acepta el Modelo Curricular por } \\
\text { Competencia }\end{array}$ & Total \\
\cline { 2 - 3 } & $\mathbf{S i}$ & No & \\
\hline Si & 74 & 0 & 74 \\
No & 35 & 20 & 55 \\
Total & 109 & 20 & $\mathbf{1 2 9}$ \\
\hline
\end{tabular}




\begin{tabular}{lc}
\hline \multicolumn{2}{c}{ Estadísticas de la Regresión } \\
\hline Coeficiente de correlación múltiple & 0.492 \\
Coeficiente de determinación $\mathrm{R}^{\wedge} 2$ & 0.242 \\
$\mathrm{R}^{\wedge} 2$ ajustado & 0.218 \\
Error típico & 0.322 \\
Observaciones & 129 \\
\hline
\end{tabular}

\begin{tabular}{lccccc}
\hline ANÁLISIS DE VARIANZA & & & \\
\hline & $\begin{array}{c}\text { Grados de } \\
\text { libertad }\end{array}$ & $\begin{array}{c}\text { Suma de } \\
\text { cuadrados }\end{array}$ & $\begin{array}{c}\text { Promedio de } \\
\text { los cuadrados }\end{array}$ & F & $\begin{array}{c}\text { Valor critico } \\
\text { de F }\end{array}$ \\
\hline Regresión & 1 & 1.028 & 1.028 & 9.916 & $\mathbf{0 . 0 0 0 4}$ \\
Residuos & 128 & 3.214 & 0.104 & & \\
Total & 129 & 4.242 & & & \\
\hline
\end{tabular}

\begin{tabular}{lcccccc}
\hline Coeficientes & $\begin{array}{c}\text { Error } \\
\text { tipico }\end{array}$ & $\begin{array}{c}\text { Estadístico } \\
\mathbf{t}\end{array}$ & Probabilidad & $\begin{array}{c}\text { Inferior } \\
\mathbf{9 5 \%}\end{array}$ & $\begin{array}{c}\text { Superior } \\
\mathbf{9 5 \%}\end{array}$ \\
\hline Intercepción & 0.000 & 0.0 & 0.000 & 1.000 & 0.15 & 0.151 \\
& & 74 & & & 0.15 & \\
$\begin{array}{l}\text { Responde } \\
\text { alas exigencias } \\
\text { del mercado } \\
\text { laboral }\end{array}$ & 0.357 & 0.1 & 3.149 & 0.004 & $\begin{array}{c}0.12 \\
6\end{array}$ & 0.588 \\
\hline
\end{tabular}

La prueba de Fisher demuestra que si existe relación entre aceptar el modelo curricular por competencias y que el modelo curricular por competencias responde a las exigencias del mercado laboral.

Se observa que el valor critico de $F$ ( $p$-valué) es menor al $\alpha=0.05$

Con una correlación alta de 0.492

\section{Correlaciones:}

\begin{tabular}{|c|c|c|c|c|c|c|c|c|c|c|}
\hline & $\begin{array}{c}\text { Pre } \\
\text { g. } \\
1\end{array}$ & $\begin{array}{c}\text { Pre } \\
\text { g. } \\
2\end{array}$ & $\begin{array}{c}\text { Pre } \\
\text { g. } \\
3\end{array}$ & $\begin{array}{c}\text { Pre } \\
\text { g. } \\
4\end{array}$ & $\begin{array}{c}\text { Pre } \\
\text { g. } \\
5\end{array}$ & $\begin{array}{c}\text { Pre } \\
\text { g. } \\
6\end{array}$ & $\begin{array}{c}\text { Pre } \\
\text { g. } \\
7\end{array}$ & $\begin{array}{c}\text { Pre } \\
\text { g. } \\
8\end{array}$ & $\begin{array}{c}\text { Pre } \\
\text { g. } \\
9\end{array}$ & $\begin{array}{c}\text { Pre } \\
\text { g. } \\
10\end{array}$ \\
\hline $\begin{array}{c}\text { Pre } \\
\text { g. } \\
1\end{array}$ & $\begin{array}{l}10 \\
00\end{array}$ & & & & & & & & & \\
\hline $\begin{array}{c}\text { Pre } \\
\text { g. } \\
2\end{array}$ & $\begin{array}{c}- \\
0.3 \\
83\end{array}$ & $\begin{array}{l}1.0 \\
00\end{array}$ & & & & & & & & \\
\hline $\begin{array}{c}\text { Pre } \\
\text { g. } \\
3\end{array}$ & $\begin{array}{l}0.4 \\
92\end{array}$ & $\begin{array}{c}- \\
0.4 \\
98\end{array}$ & $\begin{array}{l}1.0 \\
00\end{array}$ & & & & & & & \\
\hline $\begin{array}{c}\text { Pre } \\
\text { g. } \\
4\end{array}$ & $\begin{array}{l}0.4 \\
57\end{array}$ & $\begin{array}{c}- \\
0.4 \\
61\end{array}$ & $\begin{array}{c}0.6 \\
35\end{array}$ & $\begin{array}{l}1.0 \\
00\end{array}$ & & & & & & \\
\hline $\begin{array}{c}\text { Pre } \\
\text { g. } \\
5\end{array}$ & $\begin{array}{l}0.0 \\
57\end{array}$ & $\begin{array}{l}0.1 \\
44\end{array}$ & $\begin{array}{c}- \\
0.1 \\
92\end{array}$ & $\begin{array}{l}0.0 \\
89\end{array}$ & $\begin{array}{l}1.0 \\
00\end{array}$ & & & & & \\
\hline $\begin{array}{c}\text { Pre } \\
\text { g. } \\
6\end{array}$ & $\begin{array}{c}- \\
0.0 \\
42\end{array}$ & $\begin{array}{l}- \\
0.1 \\
60\end{array}$ & $\begin{array}{l}0.2 \\
30\end{array}$ & $\begin{array}{l}- \\
0.0 \\
65\end{array}$ & $\begin{array}{c}- \\
0.0 \\
42\end{array}$ & $\begin{array}{l}1.0 \\
00\end{array}$ & & & & \\
\hline $\begin{array}{c}\text { Pre } \\
\text { g. } \\
7\end{array}$ & $\begin{array}{l}0.4 \\
63\end{array}$ & $\begin{array}{c}- \\
0.3 \\
22\end{array}$ & $\begin{array}{c}0.4 \\
48\end{array}$ & $\begin{array}{l}0.5 \\
90\end{array}$ & $\begin{array}{c}0.2 \\
93\end{array}$ & $\begin{array}{c}- \\
0.0 \\
90\end{array}$ & $\begin{array}{l}1.0 \\
00\end{array}$ & & & \\
\hline $\begin{array}{c}\text { Pre } \\
\text { g. } \\
8\end{array}$ & $\begin{array}{c}0.5 \\
98\end{array}$ & $\begin{array}{c}- \\
0.4 \\
01\end{array}$ & $\begin{array}{c}0.5 \\
64\end{array}$ & $\begin{array}{c}0.6 \\
53\end{array}$ & $\begin{array}{l}0.0 \\
60\end{array}$ & $\begin{array}{c}- \\
0.2 \\
50\end{array}$ & $\begin{array}{l}0.7 \\
75\end{array}$ & $\begin{array}{l}1.0 \\
00\end{array}$ & & \\
\hline $\begin{array}{c}\text { Pre } \\
\text { g. } \\
9\end{array}$ & $\begin{array}{l}0.0 \\
57\end{array}$ & $\begin{array}{c}- \\
0.2 \\
08\end{array}$ & $\begin{array}{c}- \\
0.0 \\
21\end{array}$ & $\begin{array}{l}0.0 \\
89\end{array}$ & $\begin{array}{l}0.0 \\
57\end{array}$ & $\begin{array}{l}0.1 \\
55\end{array}$ & $\begin{array}{l}0.2 \\
93\end{array}$ & $\begin{array}{c}- \\
0.1 \\
20\end{array}$ & $\begin{array}{l}1.0 \\
00\end{array}$ & \\
\hline $\begin{array}{c}\text { Pre } \\
\text { g. } \\
10\end{array}$ & $\begin{array}{l}0.4 \\
92\end{array}$ & $\begin{array}{c}- \\
0.3 \\
71\end{array}$ & $\begin{array}{l}0.5 \\
04\end{array}$ & $\begin{array}{c}0.6 \\
35\end{array}$ & $\begin{array}{l}0.1 \\
50\end{array}$ & $\begin{array}{c}- \\
0.0 \\
56\end{array}$ & $\begin{array}{l}0.8 \\
17\end{array}$ & $\begin{array}{l}0.8 \\
24\end{array}$ & $\begin{array}{l}0.1 \\
50\end{array}$ & $\begin{array}{l}1.0 \\
00\end{array}$ \\
\hline
\end{tabular}

También se observa otras variables correlacionas y/o existencia de Relación.

\section{DISCUSIÓN}

Los docentes de la Universidad Nacional "José Faustino Sánchez Carrión" que dictan los primeros ciclos están aceptando el nuevo modelo curricular por competencias.

\section{CONCLUSIÓN}

Los docentes de la Universidad Nacional "José Faustino Sánchez Carrión" que dictan los primeros ciclos es decir con el Plan Curricular por Competencias están aceptando este nuevo modelo curricular.

\section{AGRADECIMIENTO}

Se agradece a los docentes que apoyaron en la aplicación de las encuestas.

\section{REFERENCIAS BIBLIOGRÁFICAS}

L, Yves (2010). El Enfoque por Competencias y Profesionalización de la Enseñanza: una clarificación conceptual. Revista Iberoamericana sobre Calidad, Eficacia y Cambio en Educación Volumen 9, Número 1. Madrid.

H, Bárbara (2009). Efectividad del Desempeño Docente: una reseña de la literatura internacional y su relevancia para mejorar la educación en América Latina. PREAL. Santiago de Chile.

A, Orvelis( 2006). El Proceso Pedagógico en la Educación Técnica y Profesional: antecedentes históricos, esencia y caracterización en el siglo XXI. Editorial Universidad Pedagógica Frank País García. Santiago de Cuba.

A, Félix (2001). Teoría Sinérgica del Aprendizaje en la Educación Superior. 1ra edición. Editorial: Paidos, Buenos Aires.

A. Beatrice (1999). Las Instituciones Formadoras de Docentes y las Claves para Formar Buenos Docentes, Santiago de Chile. Disponible en: 6.http://sicevaes.csuca.org/attachments/134_Las\%20Instit uciones $\% 2$ Formadoras $\% 20$ de $\% 20$ Docentes $\% 20 y \% 2$ Ol as\%20Claves\%20para\%20Formar\%20Buenos.PDF (25 de mayo 2011). 